\title{
RAdio Emission From Weak SPHERICAL SHOCKS IN THE OUTSKIRTS OF GalaXY CluSters
}

\author{
HYESUNG KANG \\ Department of Earth Sciences, Pusan National University, Pusan 609-735, Korea; hskang@pusan.ac.kr \\ Received February 9, 2015; accepted March 20, 2015
}

\begin{abstract}
In Kang (2015) we calculated the acceleration of cosmic-ray electrons at weak spherical shocks that are expected to form in the cluster outskirts, and estimated the diffuse synchrotron radiation emitted by those electrons. There we demonstrated that, at decelerating spherical shocks, the volume integrated spectra of both electrons and radiation deviate significantly from the test-particle power-laws predicted for constant planar shocks, because the shock compression ratio and the flux of inject electrons decrease in time. In this study, we consider spherical blast waves propagating through a constant density core surrounded by an isothermal halo with $\rho \propto r^{-n}$ in order to explore how the deceleration of the shock affects the radio emission from accelerated electrons. The surface brightness profile and the volumeintegrated radio spectrum of the model shocks are calculated by assuming a ribbon-like shock surface on a spherical shell and the associated downstream region of relativistic electrons. If the postshock magnetic field strength is about 0.7 or $7 \mu \mathrm{G}$, at the shock age of $\sim 50 \mathrm{Myr}$, the volume-integrated radio spectrum steepens gradually with the spectral index from $\alpha_{\text {inj }}$ to $\alpha_{\text {inj }}+0.5$ over $0.1-10 \mathrm{GHz}$, where $\alpha_{\text {inj }}$ is the injection index at the shock position expected from the diffusive shock acceleration theory. Such gradual steepening could explain the curved radio spectrum of the radio relic in cluster A2266, which was interpreted as a broken power-law by Trasatti et al. (2015), if the relic shock is young enough so that the break frequency is around $1 \mathrm{GHz}$.
\end{abstract}

Key words: acceleration of particles — cosmic rays — galaxies: clusters: general — shock waves

\section{INTRODUCTION}

Cosmological hydrodynamic simulations have shown that shock waves may form due to supersonic flows in the baryonic intracluster medium (ICM) during the formation of the large scale structure in the Universe (e.g., Ryu et al. 2003; Kang et al. 2007; Vazza et al. 2009; Skillman et al. 2011). The time evolution of such shocks in numerical simulations (e.g., Vazza et al. 2012) indicates that shock surfaces behave like spherical bubbles blowing out from the cluster center during major episodes of mergers or infalls from adjacent filaments. Shock surfaces could last for the order of the dynamical time scale of clusters, $t_{\mathrm{dyn}} \sim 1$ Gyr. This implies that some cosmological shock waves are associated with merger induced outflows, and hence shock bubbles are likely to expand into the cluster outskirts where the density profile decreases with radial distance from the cluster center.

Observational evidence for shock bubbles can be found in the so-called "radio relics" detected in the outskirts of galaxy clusters, which are interpreted as diffuse synchrotron emitting structures containing relativistic electrons accelerated at weak ICM shocks $\left(M_{s} \sim 2-4\right)$ (e.g., van Weeren et al. 2010, 2011; Nuza et al. 2012; Feretti et al. 2012; Brüggen et al. 2012; Brunetti \& Jones 2014). Some radio relics, for instance, the "Sausage relic" in cluster CIZA J2242.8+5301 and the "Toothbrush" relic in cluster 1RXS J0603.3+4214,

Corresponding Author: H. Kang have thin arc-like shapes of $\sim 50 \mathrm{kpc}$ in width and $\sim 1-2 \mathrm{Mpc}$ in length (van Weeren et al. 2010, 2012). They could be represented as a ribbon-like structure on a spherical shell and the associated downstream volume of radio-emitting electrons, projected onto the sky plane (e.g., van Weeren et al. 2010; Kang et al. 2012).

As shown in Figure 1, the viewing depth of a relic shock structure can be parameterized by the extension angle $2 \psi$, while the physical width of the postshock volume of radiating electrons is mainly determined by the advection length, $\Delta l_{\text {adv }} \approx u_{2} \cdot t_{\text {age }}$, for low energy electrons or the cooling length, $\Delta l_{\text {cool }}\left(\gamma_{e}\right) \approx u_{2} \cdot t_{\text {rad }}\left(\gamma_{e}\right)$ for high energy electrons. Here $u_{2}$ is the flow speed behind the shock and $t_{\mathrm{rad}}\left(\gamma_{e}\right)$ is the radiative cooling time scale for electrons with Lorentz factor, $\gamma_{e}$. It remains largely unknown how such a ribbon-like structure can be formed by the spherical outflows in galaxy clusters. We note, however, a recent study by Shimwell et al. (2015) who suggested that the uniform arc-like shape of some radio relics may trace the underlying region of pre-existing seed electrons remaining from an old radio lobe.

According to diffusive shock acceleration (DSA) theory, cosmic-ray (CR) particles can be generated via Fermi 1st order process at collisionless shocks (Bell 1978; Drury 1983; Malkov \& Drury 2001). In the testparticle regime, the CR energy spectrum at the position of the shock has a power-law form, $N(E) \propto E^{-s}$, where $s=(\sigma+2) /(\sigma-1)$ and $\sigma=\rho_{2} / \rho_{1}$ is the shock compression ratio. Hereafter, we use the sub- 

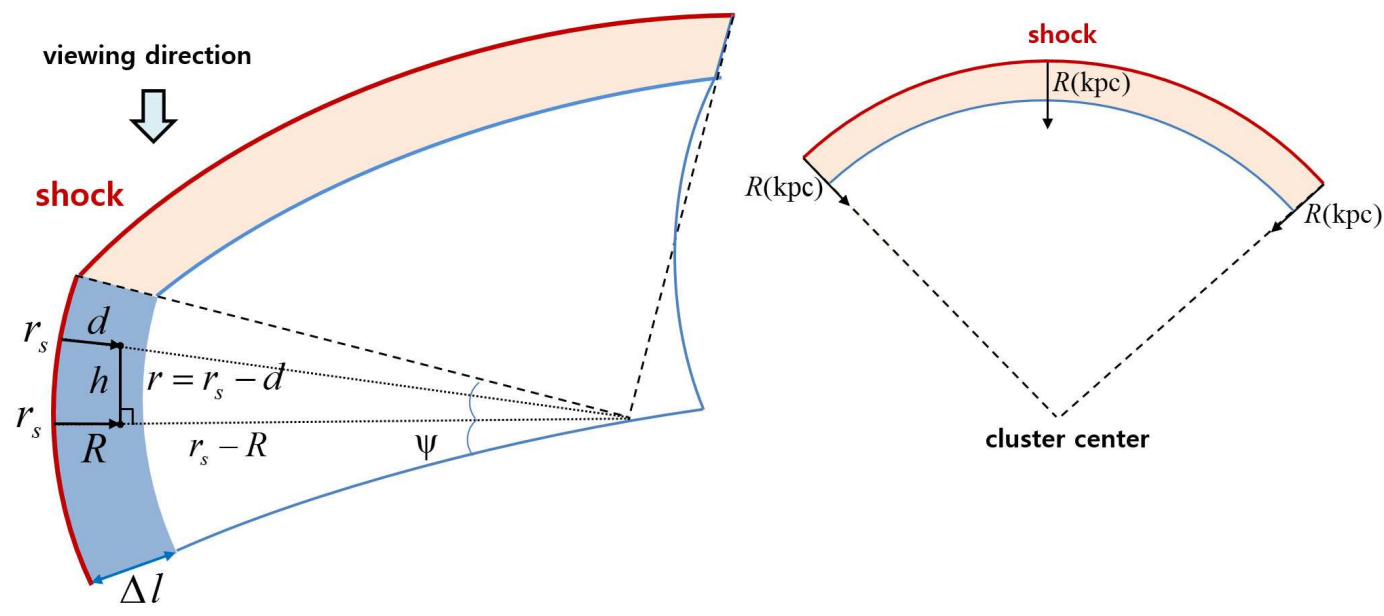

Figure 1. Left: Schematic diagram showing a spherical shock surface (red) and the postshock electron distribution (blue) behind the shock. The shock surface is modeled as a Mpc-long ribbon on the spherical surface with $r_{s}$, whose viewing depth can be defined by the extension angle $2 \psi$. The width of the postshock electron distribution behind the shock is determined by $\Delta l\left(\gamma_{e}\right) \approx u_{2} \cdot \min \left[\mathrm{t}_{\mathrm{age}}, \mathrm{t}_{\mathrm{rad}}\left(\gamma_{\mathrm{e}}\right)\right]$. The peach surface faces the observer. The surface brightness is calculated by integrating the volume emissivity $j_{\nu}(d)$ along the path length, $h$, at a given line of sight (see Equation 4 ). Here $R$ is the distance behind the projected shock edge in the plane of the sky and $d=r_{s}-r$ is the distance of a shell behind the shock, so $\left(r_{s}-d\right)^{2}=\left(r_{s}-R\right)^{2}+h^{2}$. Right: Radio image projected onto the sky plane, if viewed from the top as indicated here. The surface brightness is uniform along the thin arc-like shape as in the case of the Sausage relic in CIZA J2242.8+5301 (van Weeren et al. 2010), while it varies with the downstream distance, $R(\mathrm{kpc})$, as shown in Figures 2 and 4 .

scripts ' 1 ' and ' 2 ' to denote the conditions upstream and downstream of shock, respectively. Then the synchrotron radiation spectrum due to these CR electrons has a power-law form of $j_{\nu}\left(x_{s}\right) \propto \nu^{-\alpha_{\text {inj }}}$, where $\alpha_{\text {inj }}=(s-1) / 2=\left(M_{s}^{2}+3\right) / 2\left(M_{s}^{2}-1\right)$ is the injection index at the shock. Moreover, the volumeintegrated synchrotron spectrum downstream of a planar shock becomes a simple power-law of $J_{\nu} \propto \nu^{-A_{\nu}}$ with $A_{\nu}=\alpha_{\text {inj }}+0.5$ above a break frequency, since electrons cool via synchrotron and inverse-Compton (iC) losses behind the shock (e.g., Kang 2011). Such spectral characteristics are commonly used to infer the shock Mach number of observed radio relics (e.g., van Weeren et al. 2010; Stroe et al. 2014)

In Kang (2015) (Paper I), we calculated the electron acceleration at spherical shocks similar to Sedov-Taylor blast waves with $M_{s} \sim 2.5-4.5$, which expand into a hot uniform ICM. We found that the electron energy spectrum at the shock location reaches a steady state defined by the instantaneous shock parameters. Hence the spatially resolved, synchrotron radiation spectra at the shock can be described properly by the test-particle DSA predictions for steady planar shocks. However, the volume integrated spectra of both electrons and radiation evolve differently from those of planar shocks and exhibit some nonlinear signatures, depending on the time-dependence of the shock parameters. For instance, the shock compression ratio $\sigma$ and the injection flux of CR electrons decrease, as the shock expands and slows down, resulting in some curvatures in both electron and radiation spectra.

Magnetic fields play key roles in DSA at collisionless shocks and control the synchrotron cooling and emission by relativistic electrons. The observed magnetic field strength is found to decrease from $\sim 1-10 \mu \mathrm{G}$ in the core region to $\sim 0.1-1 \mu \mathrm{G}$ in the periphery of clusters (Feretti et al. 2012). On the other hand, it is well established that magnetic fields can be amplified via resonant and non-resonant instabilities induced by CR protons streaming upstream of strong shocks (Bell 1978; Lucek \& Bell 2000; Bell 2004). Recently, Caprioli \& Spitkovsky (2014) have shown that the magnetic field amplification (MFA) factor scales with the Alfvénic Mach number, $M_{A}$, and the $\mathrm{CR}$ proton acceleration efficiency as $\langle\delta B / B\rangle^{2} \sim 3 M_{A}\left(P_{\mathrm{cr}, 2} / \rho_{1} u_{s}^{2}\right)$. Here $\delta B$ is the turbulent magnetic fields perpendicular to the mean background magnetic fields, $\rho_{1}$ is the upstream gas density, and $P_{\mathrm{cr}, 2}$ is the downstream CR pressure. For typical cluster shocks with $2 \lesssim M_{s} \lesssim 5$ and $10 \lesssim M_{A} \lesssim 25$ (Ryu et al. 2003), the MFA factor due to the streaming stabilities is expected be rather small but not negligible, $\langle\delta B / B\rangle^{2} \sim 0.3-3$. However, it has not yet been fully understood how magnetic fields may be amplified both upstream and downstream of a weak shock in high beta ICM plasmas with $\beta_{p}=P_{g} / P_{B} \sim 100$.

Therefore, in Paper I, we considered several models with decaying postshock magnetic fields, in which the downstream magnetic field, $B_{d}(r)$, decreases behind the shock with a scale height of 100-150 kpc. Varying $B_{d}(r)$ profiles were found to have little impact on the spatial distribution of the electron energy spectrum, because the baseline cooling rate of electrons is set by $\mathrm{iC}$ scattering off the cosmic background photons. Further, electrons in a broad range of $\gamma_{e}$ contribute to $j_{\nu}(r)$, which smooths out any spatial variations. We note, however, that the $B^{2}$ dependence of the synchrotron emissivity $\left(j_{\nu} \propto N_{e} B^{2}\right)$ can become significant in some 
cases. Moreover, any nonlinear features due to the spatial variations of $N_{e}\left(r, \gamma_{e}\right)$ and $B_{d}(r)$ are mostly averaged out, leaving only subtle signatures in the volume integrated spectrum, $J_{\nu}$.

For the case with a constant background density (e.g., MF1-3 model in Paper 1), the shock speed decreases approximately as $u_{s} \propto t^{-3 / 5}$. In fact, it has not been examined, through cosmological hydrodynamic simulations, whether these shock bubbles would accelerate or decelerate as they propagate through the cluster outskirts. In this study, we have performed additional DSA simulations, in which the initial Sedov blast wave travels through a background medium with $\rho_{u} \propto r^{-n}$, where $r$ is the radial distance from the cluster center and $n=2-4$. This effectively mimics a blast wave that expands into a constant-density core surrounded by an isothermal halo with a decreasing pressure profile. In these new runs, the spherical shock decelerates much more slowly than $u_{s} \propto t^{-3 / 5}$, and so the nonlinear effects due to the deceleration of the shock speed are expected to be reduced compared to the uniform density models considered in Paper I.

We have also calculated the projected surface brightness, $I_{\nu}$, which depends on the three dimensional structure of the shock surface and the viewing direction. Because of the curvature in the structure of the model shock, synchrotron emissions from downstream electrons with different ages contribute to the surface brightness along a given line-of-sight (i.e., projection effects). So the observed spatial profile of $I_{\nu}(R)$ is calculated by assuming the geometrical configuration described in Figure 1. We note that this model with a ribbon-like shock surface gave rise to radio flux profiles, $S_{\nu}(R)$, that were consistent with those of the the Sausage relic and the double relics in $\mathrm{Z}_{\mathrm{wCl}} \mathrm{C008.8+5215}$ (e.g., van Weeren et al. 2010, 2011; Kang et al. 2012).

In paper I, we demonstrated that the spectral index of the volume-integrated spectral index increases gradually from $A_{\nu}=\alpha_{\text {inj }}$ to $A_{\nu}=\alpha_{\text {inj }}+0.5$ over a broad frequency range, $\sim(0.1-10) \nu_{\mathrm{br}}$, where the break frequency is $\nu_{\mathrm{br}} \sim 0.4 \mathrm{GHz}$ at the shock age of about 50 Myr for postshock magnetic fields $B_{2} \sim 0.7$ or $7 \mu \mathrm{G}$ (see Equation 9). Here, we explore whether such a transition can explain the broken power-law spectra observed in the radio relic in A2256 (Trasatti et al. 2015).

In the next section we describe the numerical calculations. The DSA simulation results of blast wave models with different postshock magnetic field profiles and with different background density profiles will be discussed in Section 3. A brief summary will be given in Section 4.

\section{Numerical Calculations}

\subsection{Basic Equations}

In order to calculate DSA of CR electrons at spherical shocks, we have solved the time-dependent diffusion-convection equation for the pitch-angleaveraged phase space distribution function for CR electrons, $f_{e}(r, p, t)=g_{e}(r, p, t) p^{-4}$, in the one-dimensional
(1D) spherically symmetric geometry:

$$
\begin{aligned}
& \frac{\partial g_{e}}{\partial t}+u \frac{\partial g_{e}}{\partial r}=\frac{1}{3 r^{2}} \frac{\partial\left(r^{2} u\right)}{\partial r}\left(\frac{\partial g_{e}}{\partial y}-4 g_{e}\right) \\
& +\frac{1}{r^{2}} \frac{\partial}{\partial r}\left[r^{2} D(r, p) \frac{\partial g_{e}}{\partial r}\right]+p \frac{\partial}{\partial y}\left(\frac{b}{p^{2}} g_{e}\right),
\end{aligned}
$$

where $u(r, t)$ is the flow velocity, $y=\ln \left(p / m_{e} c\right), m_{e}$ is the electron mass, $c$ is the speed of light, and $D(r, p)$ is the spatial diffusion coefficient (Skilling 1975). We adopt a Bohm-like diffusion coefficient with a weaker non-relativistic momentum dependence

$$
D(r, p)=1.7 \times 10^{19} \mathrm{~cm}^{2} \mathrm{~s}^{-1}\left(\frac{B(r)}{1 \mu \mathrm{G}}\right)^{-1}\left(\frac{p}{m_{e} c}\right) .
$$

The cooling term $b(p)=-d p / d t$ accounts for electron synchrotron and $\mathrm{iC}$ losses, and the cooling time scale for electrons is given as

$$
t_{\mathrm{rad}}\left(\gamma_{e}\right)=\frac{p}{b(p)}=3.47 \times 10^{7} \mathrm{yr}\left(\frac{B_{\mathrm{e}}}{8.4 \mu \mathrm{G}}\right)^{-2}\left(\frac{\gamma_{e}}{10^{4}}\right)^{-1}
$$

(e.g., Kang 2011). The 'effective' magnetic field strength $B_{\mathrm{e}}^{2}=B^{2}+B_{\mathrm{rad}}^{2}$ takes account for radiative losses due to both synchrotron and $\mathrm{iC}$ processes, where $B_{\text {rad }}=3.24 \mu \mathrm{G}(1+z)^{2}$ corresponds to the cosmic background radiation at redshift $z$ (Schlickeiser 2002). In this study, we set $z=0.2$ as a reference epoch and so $B_{\text {rad }}=4.67 \mu \mathrm{G}$

Here we adopt a simple phenomenological injection model, in which the particles with $p \gtrsim p_{\text {inj }} \approx$ $5.34 m_{p} u_{s}(t) / \sigma$ are allowed to cross the shock front and get injected into the CR populations (Kang et al. 2002). For spherically expanding shocks considered here, the injection momentum decreases in time as the shock decelerates. Since we are concerned with synchrotron emission from electrons alone, so long as we do not care about the exact units of flux, we can set the electron to proton ratio $K_{e / p}=1$ in the simulations. More detailed discussion on the pre-acceleration and injection of electrons can be found in Paper I.

At weak shocks in the test-particle limit, the CR feedback becomes negligible and the background flow, $u(r, t)$, is governed by the standard gasdynamic conservation equations in $1 \mathrm{D}$ spherical coordinates (Kang \& Jones 2006). The test-particle version of CRASH (Cosmic-Ray Amr SHock) code in a comoving spherical grid was used.

\subsection{Simulation Set-up}

For the initial shock structure, we adopt a Sedov-Taylor similarity solution propagating in a uniform ICM with the following parameters: the ICM density, $n_{H, 1}=$ $10^{-3} \mathrm{~cm}^{-3}$, the ICM temperature, $T_{1}=5 \times 10^{7} \mathrm{~K}$, the initial shock radius, $r_{s, i}=0.78 \mathrm{Mpc}$, and the initial shock speed, $u_{s, i}=4.5 \times 10^{3} \mathrm{~km} \mathrm{~s}^{-1}$ with the sonic Mach number, $M_{s, i}=4.3$ at the onset of the simulations. The shock parameters change in time as 
Table 1

Model Parameters

\begin{tabular}{lrr}
\hline Model & $\rho_{u}(r)$ & $B_{d}(r)$ \\
\hline MF1 $^{\mathrm{a}}$ & $\rho_{u}=\rho_{0}$ & $B_{d}=7 \mu \mathrm{G}$ \\
MF2 & $\rho_{u}=\rho_{0}$ & $B_{d} \propto \rho(r)$ \\
MF3 & $\rho_{u}=\rho_{0}$ & $B_{d} \propto \sqrt{P(r)}$ \\
BD1 $^{\mathrm{a}}$ & $\rho_{u}=\rho_{0}$ & $B_{d}=7 \mu \mathrm{G}$ \\
BD2 & $\rho_{u} \propto r^{-2}$ & $B_{d}=7 \mu \mathrm{G}$ \\
BD2b $\rho_{u} \propto r^{-2}$ & $B_{d} \propto \sqrt{P(r)}$ \\
BD3 $\rho_{u} \propto r^{-4}$ & $B_{d}=7 \mu \mathrm{G}$ \\
BD3b & $\rho_{u} \propto r^{-4}$ & $B_{d} \propto \sqrt{P(r)}$ \\
\hline
\end{tabular}

${ }^{\text {a }}$ In fact the models MF1 and BD1 are identical.

the spherical shock expands out, depending on the upstream conditions in the cluster outskirts.

\subsection{Synchrotron Radiation}

The synchrotron emissivity, $j_{\nu}(r)$, at each shell is calculated (in units of erg $\mathrm{cm}^{-3} \mathrm{~s}^{-1} \mathrm{~Hz}^{-1} \mathrm{str}^{-1}$ ), using the electron distribution function, $f_{e}(r, p, t)$, and the magnetic field profile, $B(r, t)$, from the DSA simulations. Then the radio intensity or surface brightness, $I_{\nu}\left(\right.$ erg $\left.\mathrm{cm}^{-2} \mathrm{~s}^{-1} \mathrm{~Hz}^{-1} \mathrm{str}^{-1}\right)$, is calculated by integrating $j_{\nu}$ along the path length, $h$, as shown in Figure 1:

$$
I_{\nu}(R)=2 \int_{0}^{h_{\max }} j_{\nu}(d) d h .
$$

Here $R$ is the distance behind the projected shock edge in the plane of the sky and $d=r_{s}-r$ is the distance of a shell behind the shock, where $\left(r_{s}-d\right)^{2}=\left(r_{s}-R\right)^{2}+h^{2}$. The extension angle $\psi=10^{\circ}$ is assumed in this study.

The volume integrated emissivity, $J_{\nu}=\int j_{\nu}(r) d V$, is calculated by integrating $j_{\nu}$ over the downstream volume specified with $r_{s}$ and $\psi$, as shown in Figure 1.

The spectral indices of $j_{\nu}(d), J_{\nu}$, and $I_{\nu}(R)$ are defined as follows:

$$
\begin{aligned}
\alpha_{\nu_{i}-\nu_{i+1}}(d) & =-\frac{d \ln j_{\nu}(d)}{d \ln \nu}, \\
A_{\nu_{i}-\nu_{i+1}} & =-\frac{d \ln J_{\nu}}{d \ln \nu}, \\
B_{\nu_{i}-\nu_{i+1}}(R) & =-\frac{d \ln I_{\nu}(R)}{d \ln \nu},
\end{aligned}
$$

estimated between $\nu_{i}$ and $\nu_{i+1}$. We chose the following four frequencies at the source, $\nu_{1}=240 \mathrm{MHz}, \nu_{2}=$ $600 \mathrm{MHz}, \nu_{3}=1.4 \mathrm{GHz}$, and $\nu_{4}=3.0 \mathrm{GHz}$ in Figures 2 and 4 . Then the redshifted frequency for objects at a redshift $z$ is $\nu_{\mathrm{obs}, \mathrm{i}}=\nu_{i} /(1+z)$.

\section{DSA Simulation Results}

\subsection{Shocks with Different Magnetic Field Profiles}

As in the previous study of Kang et al. (2012), we adopt the postshock magnetic field strength $B_{2} \sim 7 \mu \mathrm{G}$ in order to model the observed width of the Sausage relic $(\Delta l \sim 55 \mathrm{kpc})$ as the cooling length of $\gamma_{e} \sim 10^{4}$ electrons $\left(\sim u_{2} t_{\mathrm{rad}}\right)$, while the preshock magnetic field strength is chosen to be $B_{1} \sim 2-3 \mu \mathrm{G}$. Since $B_{1}$ cannot be constrained directly from observations, it is adjusted so that $B_{2}$ becomes about $7 \mu \mathrm{G}$ after considering MFA or compression of the perpendicular components of magnetic fields across the shock. However, we will show in the next section that the observed width can be modeled with a weaker magnetic field of $B_{2} \sim 0.7 \mu \mathrm{G}$ (see the discussion below Equation 9).

We consider several models whose characteristics are summarized in Table 1. As described in Paper I, we adopt the following downstream magnetic field profile, $B_{d}(r)$, in $\mathbf{M F}$ models:

$$
\begin{aligned}
\text { MF1: } & B_{1}=2 \mu \mathrm{G} \& B_{2}=7 \mu \mathrm{G} . \\
\text { MF2: } & B_{1}=3 \mu \mathrm{G}, B_{2}=B_{1} \sqrt{1 / 3+2 \sigma^{2} / 3}, \\
& \& B_{d}(r)=B_{2} \cdot\left(P(r) / P_{2}\right)^{1 / 2} \text { for } r<r_{s} . \\
\text { MF3: } & B_{1}=3 \mu \mathrm{G}, B_{2}=B_{1} \sqrt{1 / 3+2 \sigma^{2} / 3}, \\
& \& B_{d}(r)=B_{2} \cdot\left(\rho(r) / \rho_{2}\right) \text { for } r<r_{s} .
\end{aligned}
$$

For these models, the upstream density and temperature are assumed to be uniform.

In Figure 2, we compare these models at the shock age of $47 \mathrm{Myr}$. The evolution of the shock, i.e., $r_{s}(t) \propto$ $t^{2 / 5}$ and $u_{s}(t) \propto t^{-3 / 5}$, is identical in these three models. Synchrotron emissivity scales with the electron energy spectrum and the magnetic field strength as $j_{\nu} \propto N_{e}\left(\gamma_{e}\right) B^{2}$. Of course, the evolution of $N_{e}\left(\gamma_{e}\right)$ in each MF model also depends on the assumed profile of $B_{d}(r)$ through DSA and synchrotron cooling. In MF1 model where $B_{d}(r)=B_{2}$, the effects of shock deceleration are dramatically revealed in the spatial distribution of $j_{\nu}(d)$ (at the low frequency of $240 \mathrm{MHz}$ ). It increases downstream because of higher shock compression and higher electron injection flux at the earlier epoch. On the other hand, at the higher frequency of $1.4 \mathrm{GHz}, j_{\nu}(d)$ decreases behind the shock due to the fast synchrotron/iC cooling of high energy electrons.

The downstream increase of $j_{\nu}(d)$ at low frequencies is softened in the models with decaying postshock magnetic fields, compared to MF1 model, because of $B^{2}$ dependence of the synchrotron emissivity. In MF3 model, in which the downstream magnetic field decreases with the gas density behind the shock, the spatial distributions of $j_{\nu}(d)$ at all three frequencies decrease downstream. Thus the downstream distribution of $j_{\nu}(d)$ depends on the shock speed evolution, $u_{s}(t)$, and the postshock $B_{d}(r)$ as well as the chosen frequency. In Paper I, we indicated that signatures imprinted on synchrotron emission, $j_{\nu}(d)$, and its volume integrated spectrum, $J_{\nu}$, due to different postshock magnetic field profiles could be rather subtle to detect. That expectation arose because we examined the behavior of $\log j_{\nu}(r)$ in paper I (see Figure 6 there), while we plot $\nu j_{\nu}(d)$ in Figure 2.

As can be seen in the lower two rows in Figure 2, the surface brightness profile, $I_{\nu}(R)$, is affected by projection effects as well as the evolution of $u_{s}(t)$ and the spatial variation of $B_{d}(r)$. For instance, the gradual increase of $I_{\nu}$ just behind the shock up to $R_{\text {inf }} \approx 15 \mathrm{kpc}$ is due to the increase of the path length, and its inflection point, $R_{\text {inf }}=r_{s}(1-\cos \psi)$, depends on the value of $\psi$. 


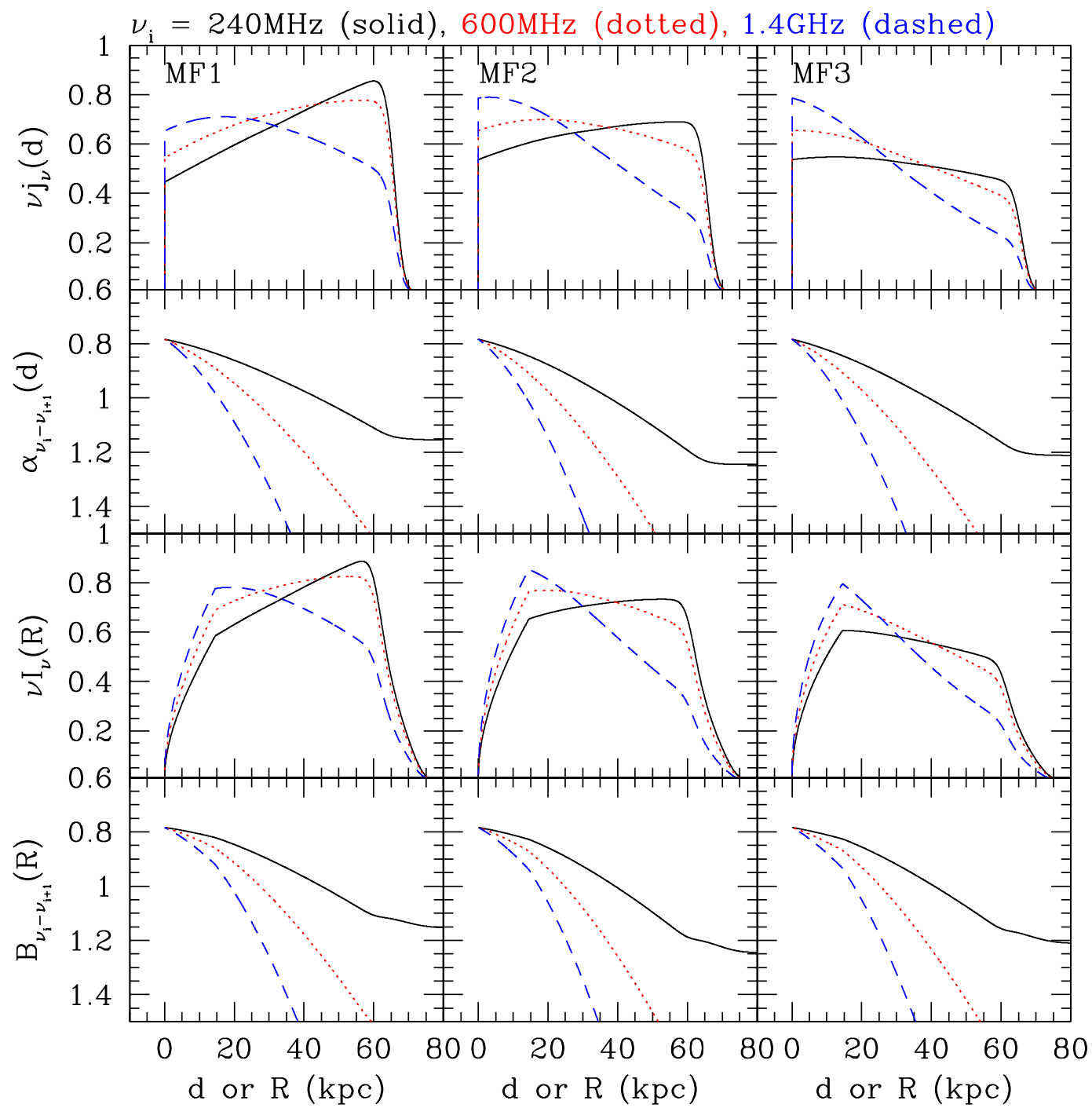

Figure 2. Spherical shock models with three different magnetic field profiles: MF1, MF2, and MF3. The results are shown at $t_{\text {age }}=47 \mathrm{Myr}$, when $r_{s}=0.96 \mathrm{Mpc}, u_{s}=3.4 \times 10^{3} \mathrm{~km} \mathrm{~s}^{-1}$ and $M_{s}=3.2$. In the upper two rows, spatial distributions of the synchrotron emissivity, $\nu_{i} j_{\nu_{i}}(r)$, and its spectral indices, $\alpha_{\nu_{i}-\nu_{i+1}(r)}$ are plotted as a function of the postshock distance $d$ from the shock surface. In the lower two rows, the intensity $\nu_{i} I_{\nu_{i}}(R)$ and its spectral indices, $B_{\nu_{i}-\nu_{i+1}}(R)$ are plotted as a function of the projected distance $R$ from the shock. The frequency is $\nu_{i}=240 \mathrm{MHz}$ (black solid line), $600 \mathrm{MHz}$ (red dotted), 1.4 GHz (blue dashed), and $3.0 \mathrm{GHz}$ for $i=1,2,3$, and 4 . The downstream volume of radio-emitting electrons is assumed to have the shape as illustrated in Figure 1 with $\psi=10^{\circ}$. Note that $j_{\nu}$ and $I_{\nu}$ are plotted in arbitrary units.

Beyond the inflection point, the path length decreases but $j_{\nu}(d)$ may increase or decrease depending on $u_{s}(t)$ and $B_{d}(r)$, resulting in a range of spatial profiles of $I_{\nu}(R)$.

Figure 2 also demonstrates that the spectral indices, $\alpha_{\nu_{i}-\nu_{i+1}}(d)$ and $B_{\nu_{i}-\nu_{i+1}}(R)$, at all three frequencies decrease behind the shock and do not show significant variations among the different $\mathbf{M F}$ models other than faster steepening of both indices for more rapidly decaying $B_{d}(r)$ profiles.

In summary, at Sedov-Taylor type spherical shocks decelerating with $u_{s} \propto t^{-3 / 5}$, the energy spectrum, $N_{e}\left(r, \gamma_{e}\right)$, of low energy electrons increases downstream behind the shock. As a result, the spatial distributions of the radio emissivity, $j_{\nu}(d)$, and the surface bright- ness, $I_{\nu}(R)$, at low radio frequencies $(<1 \mathrm{GHz})$ could depend significantly on the postshock magnetic field profile.

At high radio frequencies, such dependence becomes relatively weaker, because the width of the postshock spatial distribution of high energy electrons is much narrower. So, the magnetic field profile far downstream has less influence on synchrotron emission. On the other hand, the spectral indices, $\alpha_{\nu}$ and $B_{\nu}$, are relatively insensitive to these variations.

\subsection{Shocks with Different Background Density Profile}

In BD models, we assume that the initial blast wave propagates into an isothermal halo with a different density profile for $r>r_{s, i}$ : 

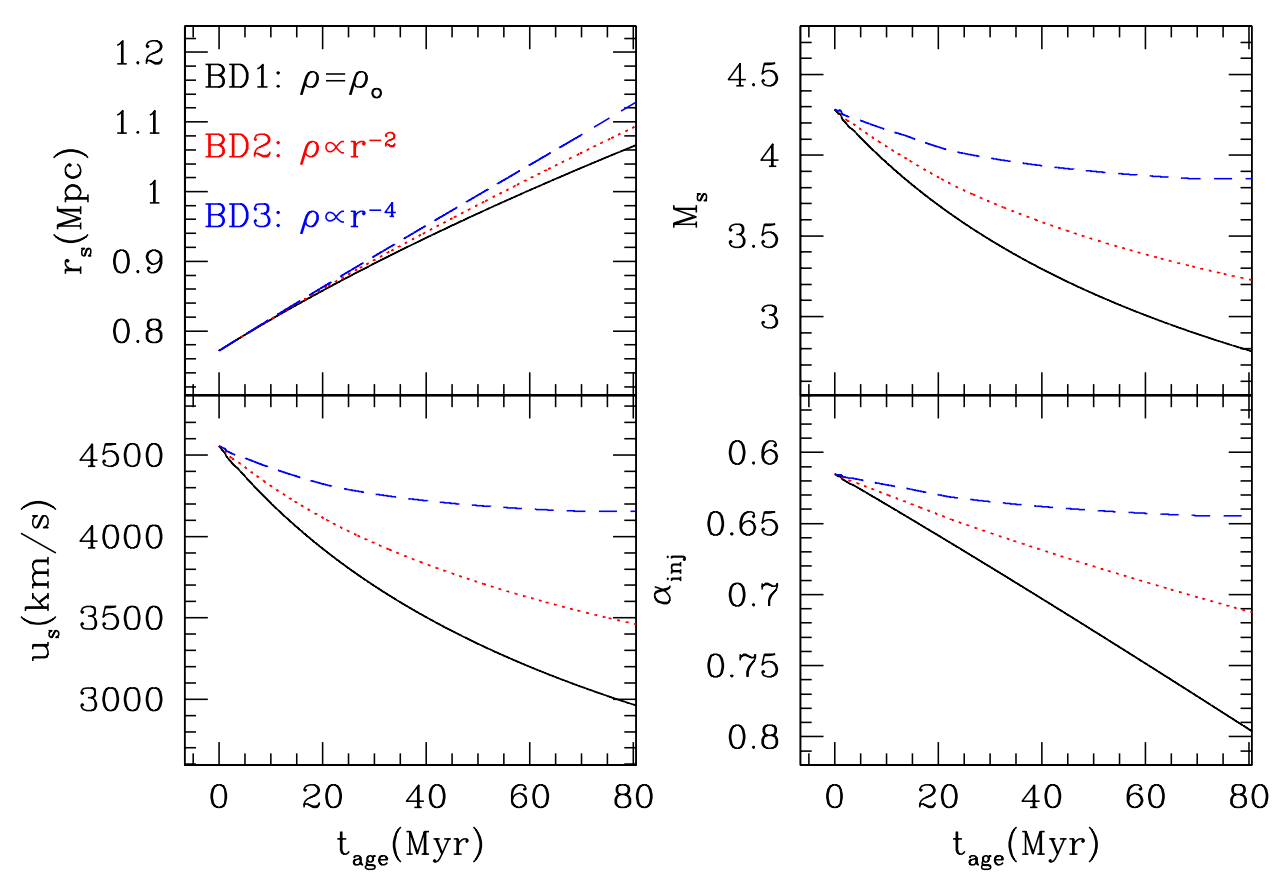

Figure 3. Evolution of spherical shock models with different background density profile, BD1 (black solid lines), BD2 (red dotted), and BD3 (blue dashed) are shown: shock radius, $r_{s}$, shock speed, $u_{s}$, sonic Mach number, $M_{s}$, and the DSA spectral index at the shock, $\alpha_{\text {inj }}$.

$$
\begin{aligned}
& \text { BD1: } \rho_{u}(r)=\rho_{0} . \\
& \text { BD2: } \rho_{u}(r)=\rho_{0} \cdot\left(r / r_{s, i}\right)^{-2} . \\
& \text { BD3: } \rho_{u}(r)=\rho_{0} \cdot\left(r / r_{s, i}\right)^{-4} .
\end{aligned}
$$

The upstream temperature is $T_{1}=5 \times 10^{7} \mathrm{~K}$, and $B_{1}=$ $2 \mu \mathrm{G}$ and $B_{2}=7 \mu \mathrm{G}$ for these models. In fact MF1 and BD1 models are identical. We also consider $\mathbf{B D 2} \mathbf{b}$ and BD3b models, in which the downstream magnetic field profile is the same as MF2, i.e., $B_{d}(r) \propto \sqrt{P(r)}$.

In the so-call beta model for isothermal ICMs, the gas distribution can be represented by $\rho(r) \propto r^{-3 \beta}$ in the outskirts of galaxy clusters (Sarazin 1988). So BD2 model corresponds to the beta model with $\beta \approx 2 / 3$, which is consistent with typical X-ray brightness profile of observed X-ray clusters. Recall that in our simulations the spherical blast wave propagating through a uniform ICM is adopted for the initial conditions. So we are effectively considering a spherical blast wave that propagates first in a constant-density core (i.e., $\rho=\rho_{0}$ for $\left.r<r_{s, i}\right)$ and then expands into a surrounding isothermal halo with $\rho \propto r^{-n}$ (for $r>r_{s, i}$ ).

With the different background density (or gas pressure) profile, the shock speed evolves differently as it expands outward. Figure 3 shows how the shock radius, $r_{s}(t)$, shock speed, $u_{s}(t)$, the sonic Mach number, $M_{s}(t)$, and the DSA spectral index at the shock position, $\alpha_{\text {inj }}$, vary in time in BD models. As expected, the shock decelerates much more slowly if the background pressure decreases outward as in BD2 and BD3 models. In BD3 model, for instance, the shock speed decreases less than $10 \%$ during 100 Myr. Hence these BD models allow us to explore the dependence of radio spectral properties for a range of the time evolution of the shock.

Figure 4 compares the surface brightness profile, $I_{\nu}(R)$, at $\nu_{1}=240 \mathrm{MHz}$ (black solid lines), $\nu_{2}=$ $600 \mathrm{MHz}$ (red dotted), and $\nu_{3}=1.4 \mathrm{GHz}$ (blue dashed) in models with different $\rho_{u}$ and $B_{d}$. In BD1 model, the shock parameters are: $r_{s}=0.83 \mathrm{Mpc}$, $u_{s}=4.1 \times 10^{3} \mathrm{~km} \mathrm{~s}^{-1}$ and $M_{s}=3.9$ at $t_{1}=13 \mathrm{Myr}$; $r_{s}=0.91 \mathrm{Mpc}, u_{s}=3.6 \times 10^{3} \mathrm{~km} \mathrm{~s}^{-1}$ and $M_{s}=3.4$ at $t_{2}=33 \mathrm{Myr} ; r_{s}=1.0 \mathrm{Mpc}, u_{s}=3.1 \times 10^{3} \mathrm{~km} \mathrm{~s}^{-1}$ and $M_{s}=2.9$ at $t_{3}=67 \mathrm{Myr}$.

The width of the radio structure at low frequencies, $\Delta l_{\text {adv }} \sim t_{\text {age }} u_{s} / \sigma$, increases with the shock age, while the amplitude of the surface brightness decreases in time (from left to right in Figure 4). For high frequencies $(>1 \mathrm{GHz})$, however, the width asymptotes to the cooling length, $\Delta l_{\text {cool }} \sim t_{\text {rad }}\left(\gamma_{e}\right) u_{s} / \sigma$. So the postshock magnetic field strength could be inferred from $t_{\mathrm{rad}}\left(\gamma_{e}\right)$ with high frequency observations. Since the synchrotron emission from monoenergetic electrons with $\gamma_{\mathrm{e}}$ in the postshock magnetic field of $B_{2}$ peaks at $\nu_{\text {peak }} \approx 0.3\left(3 e B_{2} / 4 \pi m_{e} c\right) \gamma_{e}^{2} \approx$ $0.88 \mathrm{GHz}\left(B_{2} / 7 \mu \mathrm{G}\right)\left(\gamma_{e} / 10^{4}\right)^{2}$, according to Equation (3), the cooling time scale of the electrons that emit mostly at $\nu_{\text {peak }}$ becomes

$$
\begin{gathered}
t_{\text {rad }}\left(\nu_{\text {peak }}\right) \approx \frac{\Delta l_{\text {cool }}\left(\gamma_{e}\right)}{u_{s} / \sigma}=3.26 \times 10^{7} \mathrm{yr} . \\
\left(\frac{\nu_{\text {peak }}}{1 \mathrm{GHz}}\right)^{-1 / 2}\left(\frac{B_{\mathrm{e}, 2}}{8.4 \mu \mathrm{G}}\right)^{-2}\left(\frac{B_{2}}{7 \mu \mathrm{G}}\right)^{1 / 2},
\end{gathered}
$$

where $B_{e, 2}=\sqrt{B_{2}^{2}+B_{\text {rad }}^{2}}$. Here note that the quantity, $\left(B_{e, 2} / 8.4 \mu \mathrm{G}\right)^{-2}\left(B_{2} / 7 \mu \mathrm{G}\right)^{1 / 2}$, has an identical 


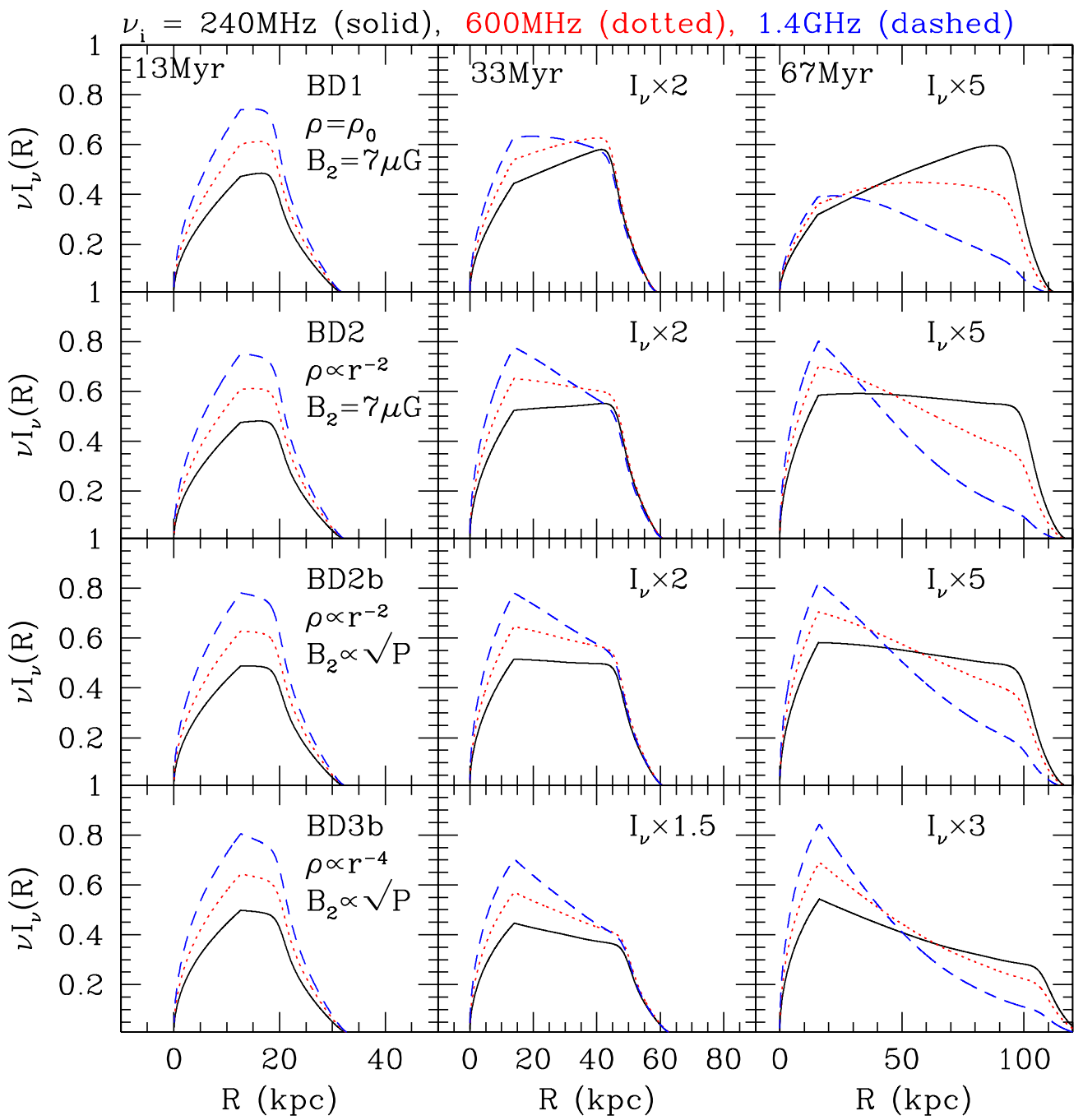

Figure 4. Spherical shock models with different background density and magnetic field profiles: BD1 (MF1), BD2, BD2b, and BD3b (from top to bottom rows). See Table 1 for the model parameters. Spatial distributions of the intensity $\nu I_{\nu}$ are shown at three shock ages, $t_{1}=13 \mathrm{Myr}, t_{2}=33 \mathrm{Myr}$, and $t_{3}=67 \mathrm{Myr}$. The frequency is $\nu_{1}=240 \mathrm{MHz}$ (black solid line), $\nu_{2}=600 \mathrm{MHz}$ (red dotted), and $\nu_{1}=1.4 \mathrm{GHz}$ (blue dashed). The downstream volume of radio-emitting electrons is assumed to have the shape as illustrated in Figure 1 with $\psi=10^{\circ}$. The intensity, $\nu I_{\nu} \cdot X$, is plotted in an arbitrary unit, where the numerical factor, $1 \leq X \leq 5$, is adopted in order to plot the quantities in the linear scale.

value for two values of $B_{2}$, e.g., 0.7 and $7 \mu \mathrm{G}$. So the downstream cooling length of the relic shocks becomes degenerate for the two possible values of $B_{2}$.

At low frequencies $(<1 \mathrm{GHz})$ the surface brightness is affected by the evolution of $u_{s}(t)$ and the spatial variation of $B_{d}(r)$ as well as the projection effects, resulting in a wide range of spatial profiles of $I_{\nu}(R)$. This suggests that, if the radio surface brightness can be spatially resolved at several radio frequencies over $\sim(0.1-10) \mathrm{GHz}$, we may extract the time evolution of $u_{s}(t)$ as well as the shock age from low frequency observations. So it would be useful to compare multifrequency radio observations with the intensity modeling that accounts for the shock evolution, magnetic field profile and projection effects.

The comparison of $I_{\nu}(R)$ of MF1 and MF2 models in Figure 2 demonstrates that the decaying postshock magnetic field could lessen the shock deceleration signatures in the surface brightness profile. If we compare $I_{\nu}(R)$ of BD2 and BD2 b models in Figure 4, on the other hand, the difference in their profiles is much smaller than that between MF1 and MF2 models. This is because the the shock deceleration effects and the ensuing downstream increase of $N_{e}\left(r, \gamma_{e}\right)$ are relatively milder in BD2 and BD2b models.

In Figure 5, we compare the volume integrated spectrum, $J_{\nu}$, and its spectral index, $A_{\nu}$, at three different shock ages, $t_{\text {age }}=13$ Myr (black solid), 33 Myr (red dotted), and 67 Myr (blue dashed) for the same set of models shown in Figure 4.

For the test-particle power-law at steady planar shocks, the radio index $A_{\nu}$ is the same as $\alpha_{\text {inj }}$ for 

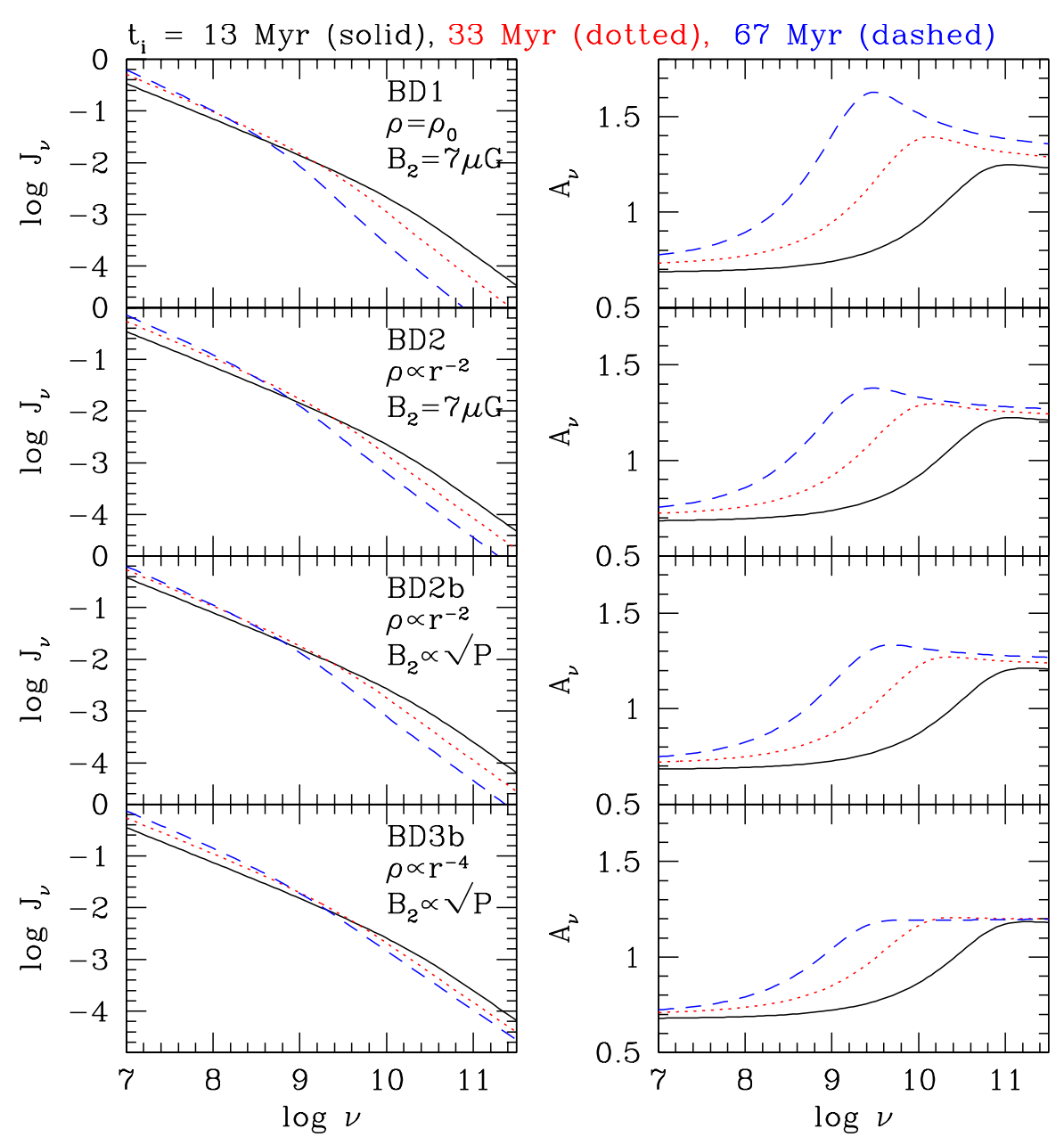

Figure 5. Same models shown as in Figure 4. Volume integrated emissivity, $J_{\nu}$, and its spectral index, $A_{\nu}$, are shown at three different shock age, $t_{1}=13 \mathrm{Myr}$ (black solid lines), $t_{2}=33 \mathrm{Myr}$ (red dotted lines), and $t_{3}=67 \mathrm{Myr}$ (blue dashed lines).

$\nu<\nu_{\mathrm{br}}$, where

$$
\nu_{\mathrm{br}} \approx 0.44 \mathrm{GHz}\left(\frac{t_{\mathrm{age}}}{50 \mathrm{Myr}}\right)^{-2}\left(\frac{B_{e, 2}}{8.4 \mu \mathrm{G}}\right)^{-4}\left(\frac{B_{2}}{7 \mu \mathrm{G}}\right)
$$

at the source. For $\nu>\nu_{\mathrm{br}}, A_{\nu}$ is expected to steepen to $\alpha_{\text {inj }}+0.5$ due to synchrotron/iC cooling of electrons. Again note that the quantity, $\left(B_{e, 2} / 8.4 \mu \mathrm{G}\right)^{-4}\left(B_{2} / 7 \mu \mathrm{G}\right)$ can have the same value for two possible value of $B_{2}$, and so the break frequency at a given shock age becomes identical as well. The observed (redshifted) break frequency corresponds to $\nu_{\mathrm{br}, \mathrm{obs}}=\nu_{\mathrm{br}} /(1+z)$. If the shock age is $t_{\text {age }} \sim 67 \mathrm{Myr}$ and $B_{2} \sim 0.7$ or $7 \mu \mathrm{G}$, for example, the observed break frequency becomes about $120 \mathrm{MHz}$ for objects at $z=0.2$. The smallest possible index is $\alpha_{\text {inj }}=0.5$ at strong shocks, so $A_{\nu}=\alpha_{\text {inj }}+0.5 \geq 1.0$ above the break frequency.

As can be seen in Figure 5, the transition from $\alpha_{\text {inj }}$ to $\alpha_{\text {inj }}+0.5$ occurs gradually over about two orders of magnitude in frequency range. If the shock is 3070 Myr old for the shock parameters considered here, the volume-integrated radio spectrum is expected to steepen gradually from $100 \mathrm{MHz}$ to $10 \mathrm{GHz}$, instead of a sharp broken power-law with the break frequency at $\nu_{\mathrm{br}, \mathrm{obs}}$. Such gradual steepening may explain why the volume-integrated radio spectra of some observed relic shocks might be interpreted as a broken power-law with $A_{\nu}<1.0$ at low frequencies.

For example, according to a recent observation of the relic in A2256, the observed spectral index is $A_{351}^{1369} \approx 0.85$ between 351 and $1369 \mathrm{MHz}$ and increases to $A_{1369}^{10450} \approx 1.0$ between 1369 and $10450 \mathrm{MHz}$ (Trasatti et al. 2015). Similar indices were obtained for our $J_{\nu}$ between 355 and $1413 \mathrm{MHz}$, and between $1413 \mathrm{MHz}$ and $10 \mathrm{GHz}$, using the DSA simulation results. The best fits are obtained for BD2b and BD3b models. In BD2b model, at $t_{\text {age }}=33 \mathrm{Myr}($ red dotted line in Figure 5 ), these spectral indices are $A_{355}^{1413} \approx 0.85$ and $A_{1413}^{10000} \approx$ 1.07. In BD3b model, again at $t_{\text {age }}=33 \mathrm{Myr}$, they are $A_{355}^{1413} \approx 0.83$ and $A_{1413}^{10000} \approx 1.03$. Thus we may explain the curvature in the integrated spectrum of the observed radio relics around $1 \mathrm{GHz}$ by the electron population radiatively cooling behind a cluster shock, if we assume that the age of the shock (or the electron acceleration duration) is about $30 \mathrm{Myr}$, which is relatively 
young compared to the dynamical time scales of typical clusters.

In the case of BD3b model, the spectral index behaves very similarly to that of a plane shock case (see Figure 4 of Paper 1), since the shock speed is more or less constant in time. Departures from the predictions for the test-particle planar shock are the most severe in BD1 (MF1) model, while it becomes relatively milder in BD2 and BD3 models with decreasing halo density profiles.

As shown in Paper I, any variations in the spatial distributions of $f_{e}(r)$ and $B_{d}(r)$ are averaged in the volume integrated quantities such as $J_{\nu}$. So signatures imprinted on the volume-integrated emission due to different $B_{d}(r)$ (e.g., between BD2 and BD2b models) would be too subtle to detect.

\section{Summary}

We have performed time-dependent DSA simulations for cosmic-ray (CR) electrons at decelerating spherical shocks with parameters relevant for weak cluster shocks: $u_{s} \approx(3.0-4.5) \times 10^{3} \mathrm{~km} \mathrm{~s}^{-1}$ and $M_{s} \approx$ $3.0-4.3$. Several models with different postshock magnetic field profiles (MF1-3) and different upstream gas density profiles (BD1-3) were considered as summarized in Table 1. Using the synchrotron emissivity, $j_{\nu}(r)$, calculated from the CR electron energy spectra at these model shocks, the radio surface brightness profile, $I_{\nu}(R)$, and the volume integrated spectrum, $J_{\nu}$, were estimated by assuming a ribbon-like shock structure described in Figure 1.

At low frequencies $(<1 \mathrm{GHz})$ the surface brightness is affected by the evolution of $u_{s}(t)$ and the spatial variation of $B_{d}(r)$ as well as projection effects, resulting in a wide range of spatial profiles of $I_{\nu}(R)$ (see Figures 2 and 4$)$. At high frequencies $(>1 \mathrm{GHz})$, for a given geometrical structure of the shock, such dependences become relatively weaker, because the width of the postshock spatial distribution of high energy electrons is much narrower and so the magnetic field profile far downstream has less influence on synchrotron emission.

For low frequency observations, the width of radio relics increases with the shock age as $\Delta l_{\text {adv }} \sim t_{\text {age }} u_{s} / \sigma$, while it asymptotes to the cooling length, $\Delta l_{\text {cool }} \sim$ $t_{\text {rad }}\left(\gamma_{e}\right) u_{s} / \sigma$, for high frequencies. If the surface brightness can be spatially resolved at multi-frequency observations over $\sim(0.1-10) \mathrm{GHz}$, we may extract significant information about the time evolution of $u_{s}(t)$, the shock age, $t_{\text {age }}$, and the postshock magnetic field strength, $B_{d}(r)$, through the detail modeling of DSA and projection effects. For instance, one may infer two possible values of the postshock magnetic field strength from the observed width of radio relics, provided that the geometrical structure (e.g., $\psi$ ) of the radio structure is known. The spectral index of $I_{\nu}(R)$, however, behaves rather similarly in all the models considered here.

If the postshock magnetic field strength is about 0.7 or $7 \mu \mathrm{G}$, at the shock age of $\sim 30 \mathrm{Myr}$, the volume- integrated radio spectrum has a break frequency, $\nu_{\mathrm{br}} \sim$ $1 \mathrm{GHz}$, and steepens gradually with the spectral index from $\alpha_{\text {inj }}$ to $\alpha_{\text {inj }}+0.5$ over the frequency range of 0.1 $10 \mathrm{GHz}$ (see Figure 5). Thus, we suggest that such a curved spectrum could explain the observed spectrum of the relic in cluster A2256 (Trasatti et al. 2015).

\section{ACKNOWLedgments}

This research was supported by Basic Science Research Program through the National Research Foundation of Korea (NRF) funded by the Ministry of Education (2012R1A1B3001065 and 2014R1A1A2057940).

\section{REFERENCES}

Bell, A. R. 1978, The Acceleration of Cosmic Rays in Shock Fronts. I, MNRAS, 182, 147

Bell, A. R. 2004, Turbulent Amplification of Magnetic Field and Diffusive Shock Acceleration of Cosmic Rays, MNRAS, 353, 550

Brüggen, M., Bykov, A., Ryu, D., \& Röttgering, H. 2012, Magnetic Fields, Relativistic Particles, and Shock Waves in Cluster Outskirts Space Sci. Rev., 166, 187

Brunetti, G., \& Jones, T. W. 2014, Cosmic Rays in Galaxy Clusters and Their Nonthermal Emission, Int. J. of Modern Physics D, 23, 30007

Caprioli, D., \& Sptikovsky, A. 2014, Simulations of Ion Acceleration at Non-relativistic Shocks. II. Magnetic Field Amplification, ApJ, 794, 46

Drury, L. O'C. 1983, An Introduction to the Theory of Diffusive Shock Acceleration of Energetic Particles in Tenuous Plasmas, Rept. Prog. Phys., 46, 973

Feretti, L., Giovannini, G., Govoni, F., \& Murgia, M. 2012, Clusters of Galaxies: Observational Properties of the Diffuse Radio Emission, A\&A Rev., 20, 54

Kang, H. 2011, Energy Spectrum of Nonthermal Electrons Accelerated at a Plane Shock, JKAS, 44, 49

Kang, H. 2015, Nonthermal Radiation from Relativistic Electrons Accelerated at Spherically Expanding Shocks, JKAS, 48, 9

Kang, H., \& Jones, T. W. 2006, Numerical Studies of Diffusive Shock Acceleration at Spherical Shocks, Astropart. Phys., 25, 246

Kang, H., Jones, T. W., \& Gieseler, U. D. J. 2002, Numerical Studies of Cosmic-Ray Injection and Acceleration, ApJ, 579, 337

Kang, H., Ryu, D., Cen, R., \& Ostriker, J. P. 2007, Cosmological Shock Waves in the Large-Scale Structure of the Universe: Nongravitational Effects, ApJ, 669, 729

Kang, H., Ryu, D., \& Jones, T. W. 2012, Diffusive Shock Acceleration Simulations of Radio Relics, ApJ, 756, 97

Lucek, S. G., \& Bell, A. R. 2000, Non-Linear Amplification of a Magnetic Field Driven by Cosmic Ray Streaming, MNRAS, 314, 65

Malkov M. A., \& Drury, L. O'C. 2001, Nonlinear Theory of Diffusive Acceleration of Particles by Shock Waves, Rep. Progr. Phys., 64, 429

Nuza, S. E., Hoeft, M., van Weeren, R. J., Gottlöber, S., \& Yepes, G. 2012, How Many Radio Relics Await Discovery?, MNRAS, 420, 2006

Ryu, D., Kang, H., Hallman, E., \& Jones, T. W. 2003, Cosmological Shock Waves and Their Role in the LargeScale Structure of the Universe, ApJ, 593, 599 
Sarazin, C. L. 1988, X-Ray Emission from Clusters of Galaxies (Cambridge: Cambridge University Press)

Schlickeiser, R. 2002, Cosmic Ray Astrophysics (Berlin: Springer)

Shimwell, T. W., Markevitch, M., Brown, S., Feretti, L, et al. 2015, Another Shock for the Bullet Cluster, and the Source of Seed Electrons for Radio Relics, MNRAS, 449, 1486

Skilling, J. 1975, Cosmic Ray Streaming. I - Effect of Alfvén Waves on Particles, MNRAS, 172, 557

Skillman, S. W., Hallman, E. J., O'Shea, W., Burns, J. O., Smith, B. D., \& Turk, M. J. 2011, Galaxy Cluster Radio Relics in Adaptive Mesh Refinement Cosmological Simulations: Relic Properties and Scaling Relationships, ApJ, 735, 96

Stroe, A., Harwood, J. J., Hardcastle, M. J., \& Rttgering, H. J. A. 2014, Spectral Age Modelling of the 'Sausage' Cluster Radio Relic, MNRAS, 455, 1213

Trasatti, M., Akamatsu, H., Lovisari, L., Klein, U., Bonafede, A., Brggen, M., Dallacasa, D., \& Clarke, T. 2015, The Radio Relic in Abell 2256: Overall Spectrum and Implications for Electron Acceleration, A\&Ap, 575, A45

van Weeren, R., Röttgering, H. J. A., Brüggen, M., \& Hoeft, M. 2010, Particle Acceleration on Megaparsec Scales in a Merging Galaxy Cluster, Science, 330, 347

van Weeren, R., Hoeft, M., Röttgering, H. J. A., Brüggen, M., Intema, H. T., \& van Velzen, S. 2011, A Double Radio Relic in the Merging Galaxy Cluster ZwCl 0008.8+5215, A\&AP, 528, A38

van Weeren, R., Röttgering, H. J. A., Intema, H. T., Rudnick, L., Brüggen, M., Hoeft, M., \& Oonk, J. B. R. 2012, The "Toothbrush-Relic": Evidence for a Coherent Linear 2-Mpc Scale Shock Wave in a Massive Merging Galaxy Cluster?, A\&AP, 546, 124

Vazza, F., Brunetti, G., \& Gheller, C. 2009, Shock Waves in Eulerian Cosmological Simulations: Main Properties and Acceleration of Cosmic Rays, MNRAS, 395, 1333

Vazza, F., Bruggen, M., Gheller, C., \& Brunetti, G., 2012, Modelling Injection and Feedback of Cosmic Rays in Grid-Based Cosmological Simulations: Effects on Cluster Outskirts, MNRAS, 421, 3375 